\title{
Adaptive Multiple Time-Scale Power Allocation for Spectrum Sharing in DS-CDMA Networks
}

\author{
Mohammad G. Khoshkholgh, Keivan Navaie \\ Department of Electrical and Computer Engineering \\ Tarbiat Modares University, Tehran, Iran \\ Email: keivan.navaie@ieee.org
}

\author{
Halim Yanikomeroglu \\ Systems and Computer Engineering Department \\ Carleton University, Ottawa, Canada \\ Email: halim@sce.carleton.ca
}

\begin{abstract}
In this paper, we propose Adaptive Multiple TimeScale Power Allocation (AMTPA) method for Opportunistic Spectrum Access (OSA) in the uplink of DS-CDMA networks. In AMTPA, the secondary service is facilitated by granting passive access to the power control commands transmitted by the primary network's base-station. AMTPA manages the secondary service transmission power in two phases, each in different time-scales. In the longer time-scale, Power Allocation Phase adaptively allocates the secondary service transmit power based on the medium-scale variations of the secondary channel, so that the achieved capacity is maximized. Then in the shorter time-scale, Power Adjustment Phase exploits the power control commands transmitted in the primary network to adaptively adjust secondary service transmission power to reduce the effect of the secondary service transmission on the Quality-of-Service of the primary network. Simulation results show that maintaining a given collision probability constraint, AMTPA makes a significant improvement on the achieved capacity.
\end{abstract}

\section{INTRODUCTION}

Spectrum Sharing was first proposed by the Federal Communications Commission (FCC) to improve the utilization of the previously allocated frequency bands [1], [2]. In spectrum sharing, an unlicensed service, namely Secondary Service, is defined which is able to access the frequency band (or a part of that) previously allocated to the licensed service, namely Primary Service. To quantify the impact of spectrum sharing on the normal operation of the primary service, Interference Temperature constraint is introduced by the FCC [3].

Generally, interference temperature constraint should specify at least two parameters [2]: the maximum interference level perceived by active primary receivers, and the maximum acceptable collision probability $\xi$. If making access to a frequency band by the secondary service does not cause the probability of crossing the interference threshold to be larger than $\xi$, the frequency band is under-utilized and is called a spectrum hole. The secondary service is permitted to make access to a spectrum hole [3]. Recently, FCC decided to abandon considering Interference Temperature for standardization purposes [4]; however for the purpose of system analysis the concept remains very applicable.

Opportunistic Spectrum Access (OSA) was proposed to opportunistically utilize the spectrum holes for the secondary service access. The idea of OSA is based on granting the allowance to the secondary service for identifying, looking for, and then exploiting the instantaneous spectrum opportunities (i.e., spectrum holes) subject to "interference temperature" constraint of the primary service.

To achieve the maximum capacity for the secondary service in OSA, prefect Channel Side Information (CSI) between the transmitter and receiver of the secondary service (secondary channel) as well as the one between the primary receiver and the secondary transmitter are required (e.g., see [5], [6]). The CSI of the channel between the primary receiver and the secondary transmitter may be fedback directly by the primary service to the secondary transmitter, or indirectly through a higher layer band manager which coordinates spectrum access [7]. Other schemes based on low rate signaling channel between the primary and the secondary systems are also proposed in [3], [8], [9]. However, because of practical reasons such scenarios based on direct cooperation between the two systems are usually difficult to implement.

The spectrum sharing over existing DS-CDMA-based networks is anticipated to be one of the spectrum sharing applications in the near future. In this paper, we propose Adaptive Multiple Time-Scale Power Allocation (AMTPA) for spectrum sharing over a DS-CDMA primary network. AMTPA tackles the issue of the availability of the CSI of the channel between the secondary transmitter and the primary receiver utilizing a multiple time-scale technique. To do this, AMTPA allocates the secondary service transmission power based on the secondary channel status in the longer timescale. It then exploits power control signaling in the shorter time-scale to adjust the secondary service transmission power based on the primary service current status. To utilize AMTPA in an existing network, the only requirement is an intersystem passive cooperation between the primary network and the secondary user. Such cooperation, in its simplest form, facilitates the secondary service by permitting to receive the power control channel of a particular primary user.

\section{System MODEL}

Fig. 1 shows the schematic diagram of the system considered in this paper. In this system, we assume a pointto-point frequency-selective Additive White Gaussian Noise (AWGN) channel. The white noise power spectral density is $N_{0}$. The channel bandwidth is $B \mathrm{~Hz}$ which is divided into $N$ flat fading $B_{c} \mathrm{~Hz}$ sub-channels, where $B_{c}$ is the channel coherence bandwidth. Sub-channels are indexed by 


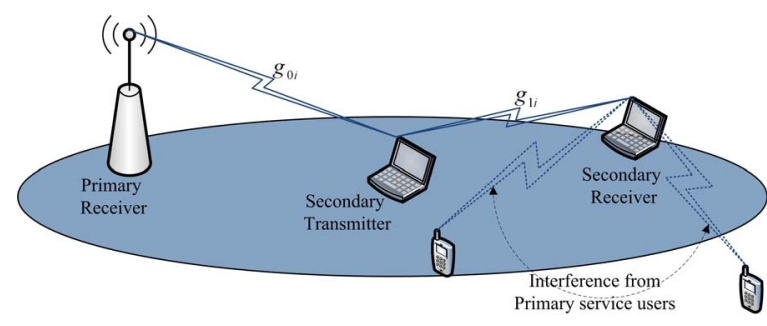

Fig. 1. System structure for a sub-channel $i$.

$i, i=1,2, \ldots, N$. The wireless channel also experiences a zero-mean Log-Normal shadowing with variance $\sigma_{d B} \mathrm{~dB}$.

Two services try to access the spectrum band: Primary Service and Secondary Service. The frequency band has been licensed to the primary service. Here, we refer to "primary spectrum" as "spectrum" unless otherwise stated. The primary network is a cellular DS-CDMA system with processing gain $G$. The secondary service does not have the spectrum license even though it may acquire access to the spectrum by adopting OSA. Subscripts $s$ and $p$ refer to the secondary service and the primary service, respectively. The maximum average transmit power of the secondary service is $\bar{P}_{s, \max }$. We assume that the secondary service (if certain conditions are satisfied) makes access to a sub-channel with $B_{c} \mathrm{~Hz}$.

The parameters $g_{0 i}, g_{1 i}$ in Fig. 1 represent the power gains of sub-channel $i$ between the secondary transmitter to the primary and secondary receivers, respectively. Both $g_{0 i}$ and $g_{1 i}$ are assumed to be stationary and ergodic independent random variables for $\forall i$ with probability density functions (pdf), $f_{0 i}\left(g_{0 i}\right)$, and $f_{1 i}\left(g_{1 i}\right)$, respectively. Perfect Channel Side Information (CSI) of the secondary channel (i.e., $g_{1 i}$ ) is available at the transmitter of the secondary service.

In the primary system a power control mechanism is adopted in the uplink so that the corresponding $E_{b} / I_{0}$ for all users in the base-station coverage area is managed to be equal to a threshold, $\rho$, which is a system parameter. In this system, the outage probability is defined by

$$
P_{\text {out }} \triangleq \operatorname{Pr}\left\{\frac{E_{b}}{I_{0}}<\rho\right\} .
$$

Time is slotted into frames, indexed by $n$, each with $T_{\text {frame }}$ seconds duration. Frame duration, $T_{\text {frame }}$, is less than or equal to the coherence time of shadowing therefore, during each frame the shadowing gain is constant. Each frame consists of $N_{p}$ slots indexed by $k, k=1,2, \ldots, N_{p}$ each with $T_{\text {slot }}$ seconds duration. The duration of time slots, $T_{\text {slot }}$, is less than or equal to the coherence time of channel fading. In this terminology, $P_{s i}(n, k)$ is the transmit power of the secondary service user at slot $k$ in frame $n$ of sub-channel $i$. We also define $I_{p \mid s}$ and $I_{s \mid p}$ as the interference imposed by the secondary service on the primary service, and the interference imposed by the primary service on the secondary service, respectively.

In the primary service the power control commands are transmitted each $T_{\text {slot }}$ seconds i.e., the power control timescale is $T_{\text {slot }}$. Power control commands are broadcasted by the base-station and received by all users (including secondary users) in the cell coverage area. For a given primary user, in the $k$ th slot of the $n$th frame, power control command $\Gamma_{p}(n, k) \in\{0,1\}$ is transmitted such that $E_{b} / I_{0}$ requirement, $\rho$, is satisfied: $\Gamma_{p}(n, k)=0\left(\Gamma_{p}(n, k)=1\right)$, corresponds to $\Delta_{p}$ Watts decrease (increase) in the current transmit power of the primary service user.

\section{OSA IN DS-CDMA PRIMARY NETWORK}

Assume that there are $M$ active primary users in the uplink of a DS-CDMA network indexed by $m, m=1,2, \ldots, M$. Without spectrum sharing, $E_{b} / I_{0}$ requirement for this system is satisfied if

$$
\frac{G Q_{m}}{\sum_{j \neq m} Q_{j}+N_{0} B} \geq \rho, \quad m=1,2, \ldots, M,
$$

where $Q_{j}$ is the received power of user $j$ at the front-end of the primary service receiver (i.e., base-station). In the ideal case in which $P_{\text {out }}=0$, the maximum number of active users in the primary network is [10]:

$$
M_{0}=\frac{G}{\rho}+1 \text {. }
$$

If $P_{\text {out }}>0$, then the number of active users in the network coverage area can be larger than that of $M_{0}$.

For a large number of users in the network coverage area, invoking the Central Limit Theorem justifies the Gaussian approximation for the interference process. Using secondorder statistics, it is shown that the interference process is also white [10]. Consequently, for a secondary service user located in the coverage area of the primary network and utilizes a $B_{c} \mathrm{~Hz}$ sub-channel, the average received interference can be approximated by

$$
I_{s \mid p}=(K-1) N_{0} B_{c} .
$$

Generally, $K \geq 1$ is a system parameter which, among other things, is a function of spatial distribution of the users and their corresponding services.

In practice, OSA is acceptable for cases in which, $M \ll M_{0}$. In such cases the secondary service utilizes OSA to access sub-channel $i$, thus imposes a narrow-band interference, $I_{i, p \mid s}$, at the receiver of the primary service (i.e., base-station). The imposed interference, $I_{i, p \mid s}$, may have impacts on the performance of the primary system. Here, we define Perfect $O S A$ as an access strategy which does not degrade the outage probability of the primary system.

Definition (Perfect OSA): A perfect OSA over sub-channel $i$ does not change $P_{\text {out }}$.

The following proposition which gives a condition on $I_{i, p \mid s}$ that results in a perfect OSA can be proved using (2).

Proposition 1: A perfect OSA over sub-channel $i$ exists if,

$$
I_{i, p \mid s} \leq G N_{0} B(K-1)\left(\frac{M_{0}}{M}-\frac{K}{K-1}\right) \triangleq Q_{I}(K) .
$$

For perfect OSA, Proposition 1 suggests that the secondary service should adjust its transmission power to keep $I_{i, p \mid s} \leq Q_{I}(K)$ in the receiver of the primary service. Proposition 1 also indicates that if the secondary service activity 
causes $I_{i, p \mid s}$ to be larger than $Q_{I}(K)$, then $E_{b} / I_{0}$ constraint is violated and an outage incident is experienced. In such cases however, the outage is a direct consequence of collision between the secondary service and the primary service transmissions.

In this paper, for brevity of discussions the collision originated outage is disjointed from the pure outage (caused by other non-spectrum-sharing related reasons). In other words, collision is an outage exclusively caused by the secondary service activity. Therefore, the collision probability, $P_{\operatorname{col}(i)}(K)$, is a metric which indicates the impact of the secondary service activity over sub-channel $i$ on the primary network performance. The acceptable level of collision probability, $\xi$, is then defined by

$$
P_{\text {col }(i)}(K)=\operatorname{Pr}\left\{I_{i, p \mid s}>Q_{I}(K)\right\} \leq \xi .
$$

Generally, $\xi$ is a system parameter set by e.g., the primary service operator or spectrum sharing regulator.

To satisfy (6), the secondary service should adjust its transmission power based on $I_{i, p \mid s}$ so that $P_{\operatorname{col}(i)}(K) \leq \xi$. However, in practice the secondary service has no direct means to estimate $I_{i, p \mid s}$. Moreover, the primary network is either not able or not particularly designed to measure and send back $I_{i, p \mid s}$ to the secondary service.

From the secondary service point of view, the base-station routinely makes reaction to violation of $E_{b} / I_{0},(5)$, by sending appropriate power control commands to the affected primary service users. Therefore, the power control commands, in a way, contains information on and about the interference condition in the receiver of the primary service. By listening to the power control commands, the secondary service might be able to adjust its transmission power such that (5) is held. In addition to (5), the secondary service should also consider the CSI of the secondary channel, $g_{1 i}$, to effectively determine its transmitted power. In this paper we suggest AMTPA to perform these two functions.

\section{Adaptive Multiple Time-Scale Power ALLOCATION (AMTPA)}

The main objective of power management in the secondary service is two-folded: 1) the secondary service transmission power should be managed such that no quality degradation is imposed on the active primary service users ${ }^{1} ; 2$ ) it should also tackle the destructive effects (or make opportunistic exploitation) of temporal variations of the secondary channel.

To achieve the first objective, the value of $I_{i, p \mid s}$ is required by the secondary service to adjust its transmission power so that the primary service $E_{b} / I_{0}$ constraint, (2), is satisfied. Generally, a common framework is required which manages both primary and secondary services and feedback $I_{i, p \mid s}$ to the secondary service transmitter. Although, as it was mentioned, considering such framework is not always attainable.

\footnotetext{
${ }^{1}$ In practice, some level of quality degradation may be acceptable by the primary service. In such cases, the secondary service should manage its power levels such that the degradation margins in the primary service is not violated.
}

As regarded to the second objective the main required input is the CSI for the secondary channel which can be easily obtained by envisaging a feedback signalling channel or a pilot channel for the secondary service.

In this paper, we propose AMTPA to implement the above mentioned two objectives. AMTPA consists of two phases each in different time-scales: Power Allocation and Power Adjustment. In Power Allocation Phase, transmit power is allocated in $T_{\text {frame }}$ time-scale with the objective of maximizing the achievable capacity of the secondary channel. Then, in Power Adjustment Phase, the allocated power is adjusted in $T_{\text {slot }}$ time-scale, such that the primary service interference constraint, (2), is satisfied.

\section{A. Power Allocation Phase}

In Power Allocation Phase, optimal power allocation for the secondary service over sub-channel $i$ is obtained with the objective of maximizing the achievable capacity of the secondary channel subject to the maximum secondary service transmission power, $\bar{P}_{s, \max }$. Therefore, the optimal secondary service power allocation is the solution of the following optimization problem:

\section{Problem $\mathcal{O}$ :}

$$
\begin{aligned}
C_{s i} & =\max _{P_{s i}} \int_{g_{1 i}} B_{c} \log \left(1+\frac{g_{1 i} P_{s i}}{K N_{0} B_{c}}\right) f_{1 i}\left(g_{1 i}\right) d g_{1 i}, \\
\text { s.t. } & \int_{g_{1 i}} P_{s i} f_{1 i}\left(g_{1 i}\right) d g_{1 i} \leq \bar{P}_{s, \max },
\end{aligned}
$$

where (7) is Shannon's capacity formula and (8) is the average transmit power constraint of the secondary service. Here, we assume that AMTPA allocates power based on the shadowing effect of the secondary channel. Problem $\mathcal{O}$ is solved at the beginning of each frame. Since fading effect of the secondary channel (if any) takes place in a shorter timescales, in AMTPA, the fading effect of the secondary channel is managed together with the primary service interference limitation in Power Adjustment Phase.

Using Lagrange's Multipliers approach, the optimal power allocation in (7) results in Water Filling (WF). The allocated power in time-slot $n$ is then obtained as follows:

$$
P_{s i, W F}(n)= \begin{cases}\frac{1}{\lambda_{0}}-\frac{K N_{0} B_{c}}{g_{1 i}(n)} & \text { if } g_{1 i}(n) \geq \lambda_{0} K N_{0} B_{c} \\ 0 & \text { O.W., }\end{cases}
$$

where $\lambda_{0}$ is Lagrangian coefficient obtained from

$$
\int_{\lambda_{0} K N_{0} B_{c}}^{\infty}\left(\frac{1}{\lambda_{0}}-\frac{K N_{0} B_{c}}{g_{1 i}}\right) f_{1 i}\left(g_{1 i}\right) d g_{1 i}=\bar{P}_{s, \max } .
$$

Substituting (9) into (7) gives the maximum achievable capacity over sub-channel $i$ for the secondary service in the Power Allocation Phase, $C_{s i}^{P A P}$,

$$
C_{s i}^{P A P}=\int_{g_{1 i} \geq \lambda_{0} K N_{0} B_{c}} B_{c} \log \left(\frac{g_{1 i}}{\lambda_{0} K N_{0} B_{c}}\right) f_{1 i}\left(g_{1 i}\right) d g_{1 i} .
$$


In AMTPA, the secondary service transmit power at the first slot of the $n$th frame, $P_{s i}(n, 1)$, is set as the output of the Power Allocation Phase, $P_{s i, W F}(n)$,

$$
P_{s i}(n, 1)=P_{s i, W F}(n) .
$$

Note that in Problem $\mathcal{O}$, the "interference temperature" constraint is not incorporated. Therefore, due to power allocation in (12), an increase on the total interference in the primary network is observed and collision incidents may be experienced by the primary service users. In the next proposition, we obtain the probability of collision, $P_{\operatorname{col}(i)}(K)$, due to performing Power Allocation Phase.

Proposition 2: If both $g_{0 i}$ and $g_{1 i}$ are Log-Normal shadowing with standard deviation $\sigma_{d B}$, the probability of collision caused by only performing Power Allocation Phase over a sub-channel $i$ is

$$
P_{c o l(i)}(K)=\frac{1}{2} \int_{\boldsymbol{\Phi}} \operatorname{erfc}\left(\frac{\ln \left[\frac{Q_{I}(K)}{\left(\frac{1}{\lambda_{0}}-\frac{K N_{0} B_{c}}{\alpha}\right)}\right]}{\sqrt{2} \sigma}\right) f_{1 i}(\alpha) d \alpha,
$$

where, $\boldsymbol{\Phi} \triangleq\left\{\alpha \mid \alpha \geq \lambda_{0} K N_{0} B_{c}\right\}, \operatorname{erfc}(x) \triangleq \frac{2}{\sqrt{\pi}} \int_{x}^{\infty} e^{-t^{2}} d t$, $f_{1 i}(x)=\frac{1}{x \sigma \sqrt{2 \pi}} \exp \left(-\frac{(\ln x)^{2}}{2 \sigma^{2}}\right)$, and $\sigma=10^{\sigma_{d B} / 10}$.

Proof: See [11].

In practice, both fading and shadowing effects are contributed in wireless channel gain. If $g_{0 i}$ consists of fading as well, for a primary network which is under-loaded (i.e., moderate number of primary users, $M$ ), on average, the destructive impact of implied interference by the secondary service power transmission on the primary service users is decreased. In such cases, $P_{\operatorname{col}(i)}(K)$ which is obtained in Proposition 2 acts as an upper-bound. Although for an over-loaded primary network (i.e., large number of users) the total interference is already in critical condition thus on average, because of the secondary service activity, the destructive impact of implied interference by the secondary service power transmission on the primary service users is increased. In such cases, $P_{\text {col }(i)}(K)$ which is obtained in Proposition 2 acts as a lower-bound.

\section{B. Power Adjustment Phase}

In AMTPA, the destructive impact of Power Allocation Phase on the collision probability as well as corresponding wireless channels' short-scale variations are then corrected in Power Adjustment Phase. Power Adjustment Phase adaptively adjusts transmission power in the shorter time-scale, i.e., $T_{\text {slot }}$ by exploiting the power control commands transmitted to a primary user $m$. Primary user $m$ is selected either randomly or by the primary network through collaboration framework with the secondary service.

In AMTPA, we set $P_{s i}(n, 1)=P_{s i, W F}(n)$, for slot $k$, $k>1$ in frame $n$. Power Adjustment Phase then adjusts the transmit power based on $\Gamma_{p}(n, k)$ so that the transmission power of the secondary service is decreased (increased) by $\Delta_{s}(n)$ watts if $\Gamma_{p}(n, k)=1\left(\Gamma_{p}(n, k)=0\right)$ :

$$
P_{s i}(n, k)=\left(P_{s i}(n, k-1)+\Delta_{s}(n)\left(1-2 \Gamma_{p}(n, k)\right)\right)^{+},
$$

where $(x)^{+}=\max \{0, x\}$.

Note that (14) is a pessimistic strategy in which it is implicitly assumed that any increase in the interference received by the primary service receiver is exclusively imposed by the secondary service access. Therefore, Power Adjustment Phase decreases the number of violation incidents of $E_{b} / I_{0}$ constraint; thus the collision probability is decreased. This can be proved as the following Proposition.

Proposition 3: Utilizing Power Adjustment Phase over subchannel $i$ based on the power control commands of user $m$ in each frame based on the allocated power by Power Allocation Phase, the achievable capacity of the secondary service is equal to $C_{s i}^{P A P}$ and

$$
P_{\operatorname{col}(i)}^{A d j}(K)<P_{\operatorname{col}(i)}(K),
$$

where $P_{c o l(i)}^{A d j}(K)$ is the collision probability of the primary service after performing Power Adjustment Phase.

Proof: See [11].

\section{Simulation Results}

We simulate a system including a primary cellular DSCDMA network and a single secondary user. The detailed simulation parameters are presented in Table I. The wireless channel modelled as Log-Normal shadowing. In some cases a Rayleigh fast fading is also considered. A fast power control mechanism is performed in the primary service network in which base-stations transmit power control commands each $5 \mathrm{~ms}$ (200 commands/s). User $m$ is selected randomly and AMTPA manages access over a given $96 \mathrm{KHz}$ sub-channel in the uplink spectrum. As the main performance measure we investigate $P_{\operatorname{col}(i)}(K)$. Since $K$ is given in our simulations hereafter, we drop $K$ for brevity.

\section{A. Impact of Power Adjustment Phase}

To show the corrective impact of the Power Adjustment Phase on the collision probability, we compare $P_{\text {col }(i)}$, for two cases. In the first case, we obtain the probability of collision for a system which only utilizes the Power Allocation Phase in the longer time-scale (i.e., $T_{\text {frame }}=160 \mathrm{~ms}$ ). In the second case, Power Adjustment Phase is also performed in a shorter timescale (i.e., $T_{s l o t}=5 \mathrm{~ms}$ ). In both cases the simulated wireless channel between the secondary transmitter and the primary receiver consists of fading as well as shadowing with the above described parameters. Fig. 2 also indicates the corrective effect of Power Adjustment Phase in significant decreasing which makes over the collision probability. As it is seen in this figure, increasing the number of users in the primary network, AMTPA collision performance in getting closer to that of one achieved by only performing Power Allocation Phase. 
TABLE I

SIMULATION PARAMETERS

\begin{tabular}{l|c}
\hline Parameter & Value \\
\hline$N_{p}$ & 32 \\
Shadowing Coherence Time & $160 \mathrm{~ms}$ \\
Fading Coherence Time & $5 \mathrm{~ms}$ \\
Cell radius & $100 \mathrm{~m}$ \\
Spectrum Bandwidth & $3.84 \mathrm{MHz}$ \\
Standard dev. of Log-Normal shadowing & $8 \mathrm{~dB}$ \\
Propagation loss exponent & 4 \\
Background noise density & $-174.0 \mathrm{dBm} / \mathrm{Hz}$ \\
$\bar{P}_{s, \text { max }}$ & $1 \mathrm{Watt}$ \\
$G$ & 128 \\
$K$ & 2 \\
$N$ & 40 (each $96 \mathrm{KHz}$ width) \\
Minimum required $E_{b} / I_{0}$ & $2 \mathrm{~dB}$ \\
$M_{0}$ & $\simeq 82$ \\
\hline
\end{tabular}

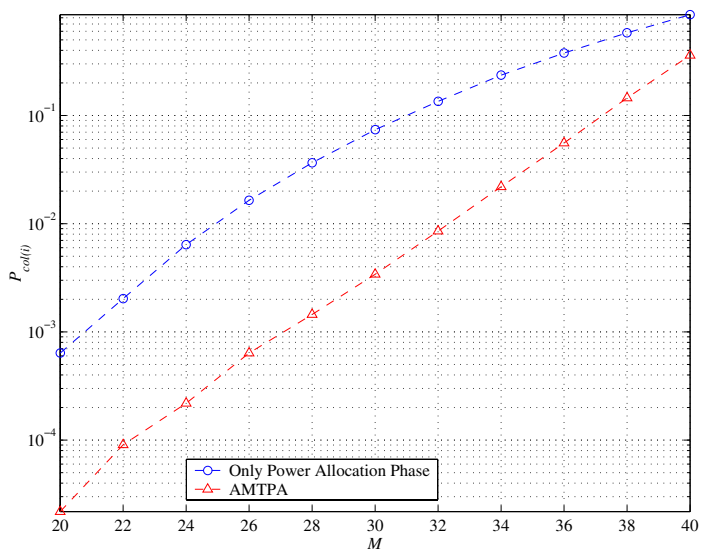

Fig. 2. Collision probability vs. $M$, for a system which only performs Power Allocation Phase and a system which also performs Power Adjustment Phase.

\section{B. Achievable Capacity}

In [5] it is shown that in cases when both $g_{0 i}$ and $g_{1 i}$ are Rayleigh fading channels the maximum achievable capacity of the secondary service is

$$
C_{0}=B_{c} \frac{\alpha \log \alpha}{\alpha-1}
$$

where $\alpha^{-1}=K N_{0} B_{c} / Q_{I}(K)$. Here we compare $C_{0}$ and the achievable capacity of AMTPA. Note that in AMTPA in addition to the fading, channels also experience shadowing. Fig. 3 indicates that without AMTPA, by increasing the number of the active primary users, the achievable capacity is reduced. This is because in [5] the collision probability is assumed to be 0 , however in AMTPA the primary service experiences a higher collision probability. For example for $M=40$, referring to Fig. 2 the collision probability is more than 0.01. In other words, using AMTPA, at the cost of a slight collision probability degradation a significant gain in achieved capacity is obtained. The maximum AMTPA achieved capacity is almost constant since in AMTPA, Power Allocation Phase allocates power based on the secondary channel medium-scale channel variations (shadowing).

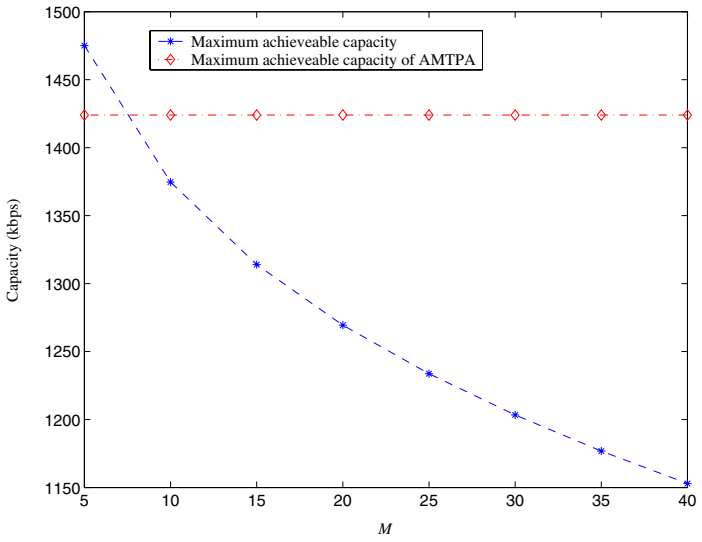

Fig. 3. Achievable capacity of the secondary service $v s$. number of users.

\section{CONCLUSIONS}

In this paper, Adaptive Multiple Time-Scale Power Allocation (AMTPA) method for Opportunistic Spectrum Access in DS-CDMA wireless networks was proposed. AMTPA conducts the secondary service transmission power management in two phases each in different time-scales. In the longer time-scale, Power Allocation Phase adaptively allocates the secondary service transmit power based on the medium-scale variations of the secondary channel. Then in the shorter time-scale, Power Adjustment Phase exploits the primary network power control commands to adjust secondary service transmission power to reduce the effect of the secondary service transmission on the Quality-of-Service of the primary network. Simulation results showed that by using AMTPA, maintaining a given collision probability constraint, significant improvement on the achieved capacity is obtained.

\section{REFERENCES}

[1] "Spectrum policy task force report," Federal Communications Commission, Tech. Rep., November 2002.

[2] Q. Zhao and B. Sadler, "A survey of dynamic spectrum access: Signal processing, networking, and regulatory policy," IEEE Signal Processing Magazine, vol. 24, no. 3, pp. 79-89, May 2007.

[3] S. Haykin, "Cognitive radio: Brain-empowered wireless communications," IEEE Journal on Selected Areas in Communications, vol. 23, no. 2, pp. 201-220, February 2005.

[4] "Establishment of an interference temperature metric to quantify and manage interference and to expand available unlicensed operation in certain fixed, mobile and satellite frequency bands (ET Docket no. 03-237)." FCC, Tech. Rep., May 2007, [online] Available: http://www.fcc.gov.

[5] A. Ghasemi and E. S. Sousa, "Fundamental limits of spectrum-sharing in fading environments," IEEE Transactions on Wireless Communications, vol. 6, no. 2, pp. 649-658, February 2007.

[6] S. Srinivasa and S. A. Jafar, "Soft sensing and optimal power control for cognitive radio," in Proceedings of the IEEE Global Communications Conference (GlobeCom'07), Washington, DC, 26-30 November 2007.

[7] J. M. Peha, "Approaches to spectrum sharing," IEEE Communications Magazine, pp. 10-12, February 2005.

[8] R. Etkin, A. Parekh, and D. Tse, "Spectrum sharing for unlicensed bands," IEEE Journal on Selected Areas in Communications, vol. 25, no. 3, pp. 517-528, April 2007.

[9] P. Stocia and T. Sundia, "On non-parametric spectral estimation," Circuits, Systems and Signal Processing, vol. 16, pp. 169-181, 1999.

[10] D. Tse and P. Viswanath, Fundamentals of Wireless Communication. Cambridge University Press, 2004.

[11] M. G. Khoshkholgh, K. Navaie, and H. Yanikomeroglu, "Multiple timescale power allocation for spectrum sharing," Preprint, January 2008. 